\title{
FPGA BASED SELF-DRIVING CAR WITH MACHINE VISION BACKGROUND
}

\author{
Reeju Elisa Baby, Leah Sibi, Lija Mariam Biju, Sahi Ann George, Shalu Shaji \\ Department of ECE \\ Amal Jyoti College, Kottayam, Kerala, India
}

\begin{abstract}
Advancement in automotive technology has got its attraction due to self-driving and electric vehicles. In this paper we are designing and Prototyping of a Fully Autonomous Car Using Machine Vision with help of Open Computer Vision software library Open CV and Xilinx FPGA. The main aim of our project is to make Fully Autonomous Car using commercially available and reliable Components and Open source Software libraries. This could save huge cost and dependency requirements. In this project we are proposing a system with Computer Vision Deep-learning that make use of a camera along with video processing unit to guide the car and make it a safer option to ride. Images captured by the camera would be sent to the real world with the help of Xilinx FPGA. By using this technology, it is made free from depending on the Custom component manufacturers and also provides a much faster time to the production level. It is very cost saving to user as affordable pricing with additional benefits of safety traffic to public.
\end{abstract}

Keywords - Raspberry Pi, FPGA, Master Control facility, Camera Module, Master- Slave, Self-Driving Car

\section{INTRODUCTION}

Globally, around 1.3 million people die in road due to crashes each year, on average 3,387 deaths a day. And talking about India the number of people who were killed in a road accident in 2013 alone were 1, 37,000 [1]. Car accidents mainly occur due to brake jam, over speed and loss of control, Control loss while driving the vehicle neutral in descent plane, damage/jam of acceleration cable, talking on a phone while driving, driving by a drunken person and driving without obeying the traffic rules. Nowadays, we could see that the drivers appear to be impatient or they would be in a hurry to reach their work places and all. People living now have little respect for traffic rules due to which at least 200 people die every day due to uncontrolled crossing on Indian road another 50 are crushed to death every day at junctions that have some sort of traffic signal or police deployment. Since the accidents increasing day by day has become an important aspect it is very essential to reduce the human error and serve the mankind. All this could come to an end with the help of self-driving cars.
Electric vehicles are facing huge drawback due to charging and replacement of batteries. The self-driving cars got huge attraction due to precision control and electrical management. A self-driving car is defined as the vehicle which is capable sensing its environment with little or no human input at all. It helps the people to take to their destination with safety as their first priority. Commencing from the early radar-based collision detection to the current days technology there has been a progress in this field. For self-driving cars, several real-time systems such as localization, route planning and movement control, environment mapping and understanding must work tightly together. These systems work together simultaneously and are self-organising system [2]. This autopilot system is supposed to have faster instinctive than human which makes better judgement causing less human error. The basic model of any autopilot system consists of a front facing camera module which can be used for environment detection i.e. to detect the traffic lights, signs pedestrians by using image processing algorithms, radars which are used for the same purpose as LIDAR. Its advantage over LIDAR is that it is light weight and can be used to calculate the velocity and speed of the vehicle. They all work in the real time environment which makes the vehicle to respond accordingly.

The image processing algorithms using software approach (MATLAB) is slower due to limited speed of the processor. This have a bad hand in real time implementation. In order to sort this problem here we make use of VLSI technology to make the processor for implementation of image processing algorithms. In recent years, development of micro electronic technology and large-scale integrated circuits manufacturing technology especially FPGA, provides new ideas and methods in order to improve the performance of image processing system. Due to the large amount of data and fast processing speed needed of the low- level image preprocessing, the image processing system based on FPGA is well used in image pre-processing field and video processing.

\section{RELATED WORK}

The project done by Gurjashan Singh Pannu et al [3], mainly focused on developing a prototype monocular vision autonomous car using Raspberry pi and the HD camera along 


\section{International Journal of Engineering Applied Sciences and Technology, 2020 \\ Vol. 5, Issue 5, ISSN No. 2455-2143, Pages 122-128 \\ Published Online September 2020 in IJEAST (http://www.ijeast.com)}

with ultrasonic sensor which provides essential data from the real world to the car. In order to provide real time computer vision, Open $\mathrm{CV}$ is being used. The proposed system makes use of existing algorithms like lane detection, obstacle detection and avoidance, these are combined together to make necessary controlling action to the car

A.M. Shah [4] paper proposes underwater images enhancement by wavelet decomposition-based image fusion implementation on FPGA.Underwater images are affected due to poor color contrast and poor visibility, which is a significant issue faced in Ocean Engineering. The objects in the image are hardly seen, not detectable and cannot be traced properly. So as a solution to this issue, here we use image fusion of multi-images of the same object into a high-quality image with abundant information. A Field Programmable Gate Array (FPGA) which is a reconfigurable hardware, that provides better features than DSP and other hardware devices due to their product fidelity and sustainable advantages in digital image and video processing, is used in this project for image processing.

Ayesh Iqbal [5] presents the model of multifunctional autonomous car using sensors. The system presented is able to track the line track, detect obstacles, take sharp bends and turns, follow traffic signals and turn on its lights under low light conditions. DC motors are used for the speed controlling action, and with help of the LDR on-off of head light take place. Stepper motor is used with the steering mechanism of the car and to take sharp turns up to 60 degrees. Ultrasonic sensors are used for detecting the nearby objects. The overall coordination and controlling action is done by the Arduino microcontroller.

In the project of Lionel Heng [6] decision making takes place using the inputs that are obtained using multiple camera which are placed at particular places of the vehicle. Here 3D perception for the proper movement of vehicle in those areas where, GPS can't be used. For the localization purpose Direct Visual-Inertial Odometry, 3-DoF Localization with Satellite Imagery, Sparse 3D Map Reconstruction, 6-DoF Localization with a Sparse 3D Map where used.

The work done by Marwan S. Alluhaidan [7] aims to improve driver safety in inclement weather but easily can be modified to enhance low vision surveillance videos as well. In this project a method to improve low visibility during bad weather conditions through the use of Retinex, which is an enhancement technique inspired by our own biological vision mechanisms is introduced.

Phanindra Amaradi et al [8] proposed his project which presents techniques developed for lane following and obstacle detection capabilities for autonomous vehicles. The proposed system is based on a single 120 degrees fish eye camera and 180 degrees scanning LIDAR sensor. For the lane detection a modified lane marking technique was used representing the position of the lines making the lane with respect to the position of the camera mounted on the vehicle. The paper describes implementation of the lane detection system using
Hough transform to detect the lanes. Based on the detected lane markings, the position of the relative vehicle is determined as to whether it is in its lane or if it has drifted outside the lane. The system shows the offset amount, as a percentage of distance, it has drifted from the center of the lane. The SICK LIDAR LMS-291 S-O5 laser range sensor is adopted for the obstacle detection.

\section{IMPLEMENTATION DETAILS}

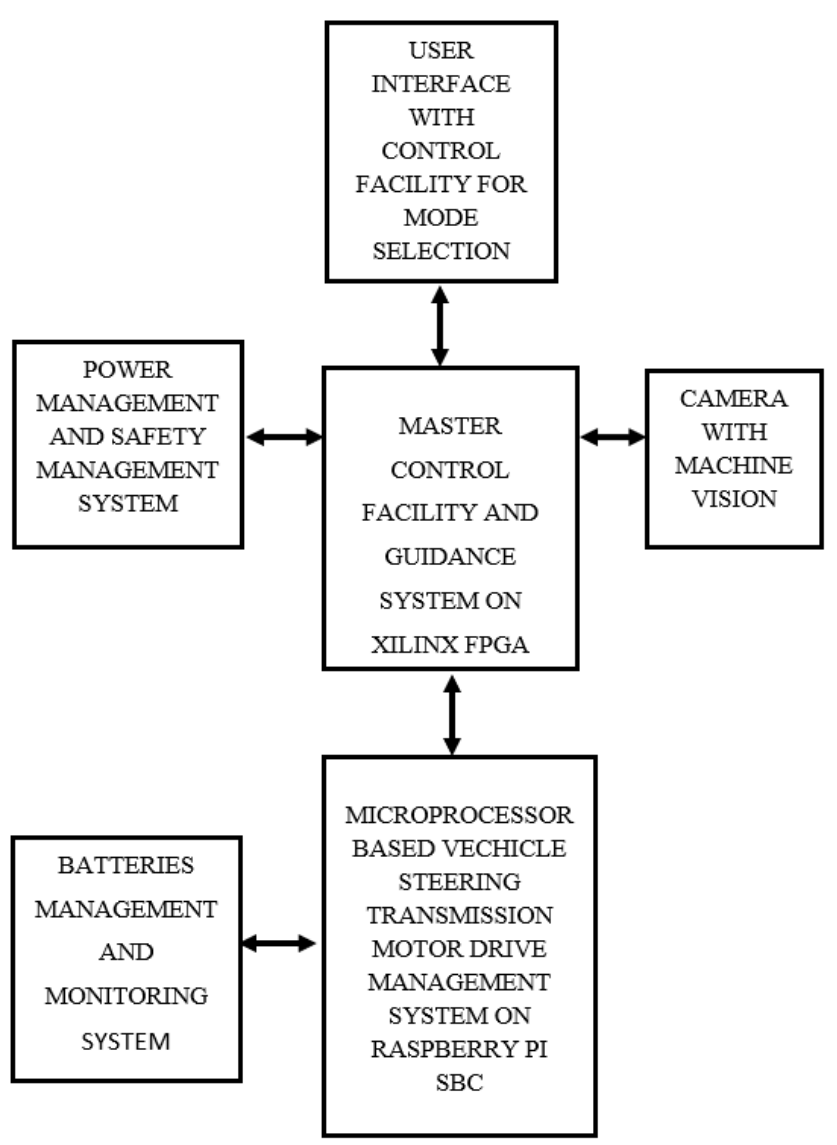

Fig. 1. Proposed self-driving model

The proposed model consists of a user interface mode selection whether to start/stop the car manually or automatically. It could be either simply turned on/ off or by interfacing an app in the mobile it could automatically turn on/off. The power management system ensures a minimum threshold of the battery for the smooth running of the vehicle. The input image is taken using a pi cam attached with Raspberry pi on the car [1]. The movement of the vehicle is controlled using the Raspberry Pi. A power bank is used for the management of Raspberry Pi. Proper safety measures are taken for the vehicle depending on the number of turns and angles taken by the vehicle. The FPGA used here is connected with a Pi cam. The Pi camera used here captures 


\section{International Journal of Engineering Applied Sciences and Technology, 2020 \\ Vol. 5, Issue 5, ISSN No. 2455-2143, Pages 122-128 \\ Published Online September 2020 in IJEAST (http://www.ijeast.com)}

live stream video which is given to the Xilinx FPGA which gives the necessary commands. Xilinx FPGA would identify the object which are trained by the program and would produce necessary command signals. These command signals are transferred to Pi board. Based on the commands from the FPGA, the Raspberry Pi generates PWM waves, i.e. the signal used for generating motor driver. This way the motor driver works. For example, if the vehicle wants to turn left, then the FPGA would give the signal to Raspberry Pi to operate the motor on the left accordingly.

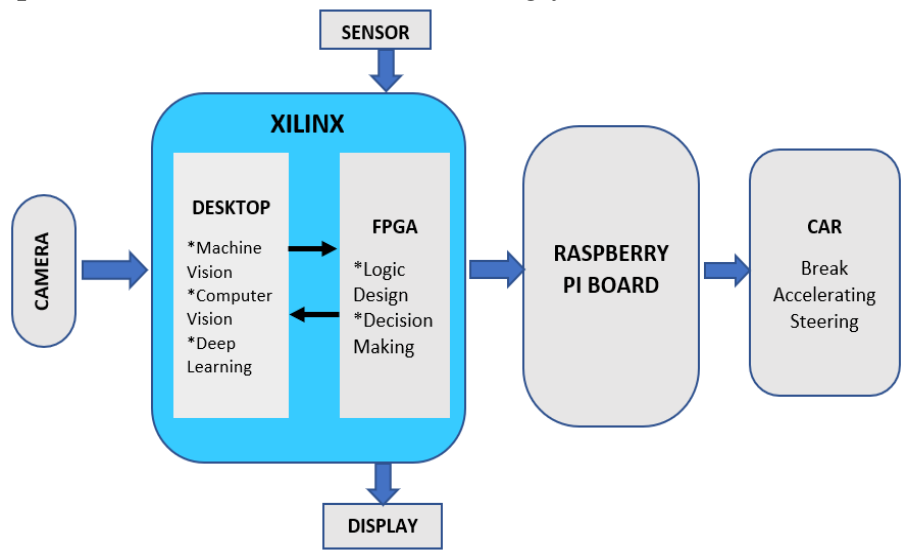

Fig. 2. Proposed self-driving model

In this project, a fully autonomous car using machine vision with the help of Open CV, FPGA and Raspberry Pi Single board computer is made. The system implementation is mainly divided into two stages. The first stage is the video capturing and machine vision technique along with Open CV which is being performed by FPGA. In the second stage, there comes the controlling action which is done by Raspberry Pi. Here we mainly use the master slave mechanism. FPGA is the master who give the command and the slave Raspberry Pi will act accordingly. From figure 2 an overall block diagram of the system could be seen . The camera module is connected to the FPGA. As the data comes to the FPGA it analyzes the video and identifies the required data and information already programmed. Here we use Python language for programming the Raspberry Pi. As per the information attained from the video, FPGA will decide the commands. The Pi board will initiate the controlling actions according to the command received and thus the system works.

\section{A. Hough Transform}

The basics of Hough transform is to find aligned points in images that creates inputs of lines i.e., Hough transform is mainly used here for lane detection within our region of interest. The vehicle in the image would turn left /right based on the curvature of the road within the frame. We know that the common factor displayed on the road is the white line, so if a vehicle wants to take a bend on the road, it is denoted by an angle $\theta$. If the vehicle is moving in a straight line, then $\theta=0$ and if the vehicle moves forward and approaches any bend then $\theta$ value keeps decreasing. When Hough transform is used in Open CV it would help to denote the $\theta$ value. It is unaffected by gaps in curves and noise.

\section{B. Hough Transform-Global Representation}

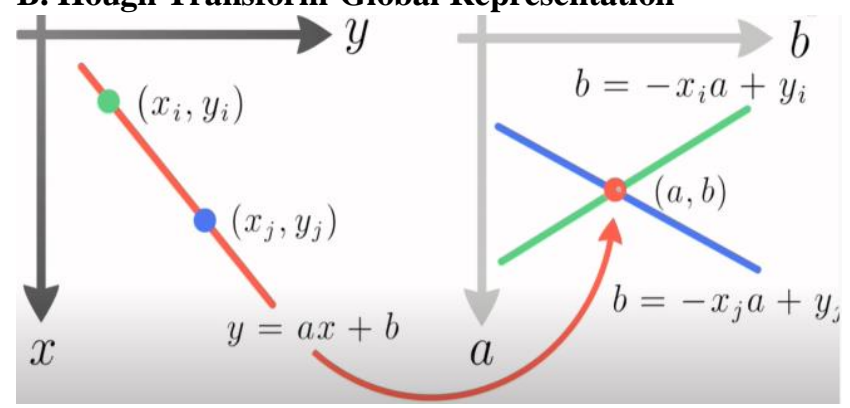

Fig. 3. Mapping one unique line to the Hough Space

Consider the $(\mathrm{x}, \mathrm{y})$ coordinate from Figure 3 where a and $\mathrm{b}$ are used to define the angulation of the line which is denoted by the line equation $y=a x+b$. This line has combination values of $\mathrm{x}$ and $\mathrm{y}$ and fixed values of $\mathrm{a}$ and $\mathrm{b}$.

Consider the plane of $a$ and $b$ which can be called as the feature space. Here the point $x_{i}$ and $y_{i}$ defines lot of possibilities on the line $\mathrm{b}=-x_{i} \mathrm{a}+y_{i}$ and the other point on the line $\mathrm{b}=-x_{\mathrm{j}} \mathrm{a}+y_{\mathrm{j}}$. When these points are intersected then $\mathrm{a}$ point of $a$ and $b$ will be defined, where the two lines match. So, if the two points are detected as edges in edge image then it would define one point here. If there are many points in $\mathrm{x}, \mathrm{y}$ plane, then all of it would match on the point $(a, b)$.

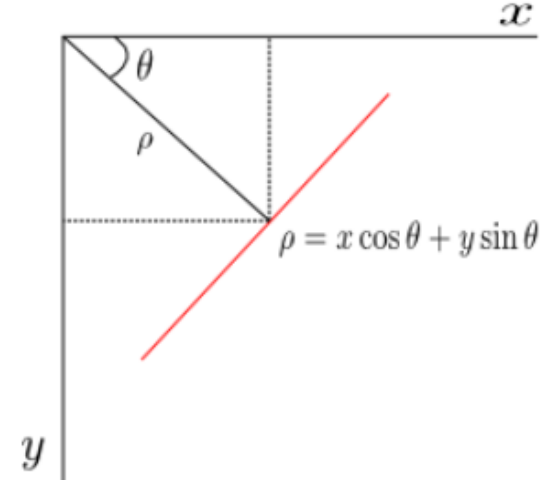

Fig. 4. Feature space in polar form

Figure 4 shows that line has two parameters i.e. the slope and intercept which represented in polar form is denoted by $(\rho, \theta)$. A straight-line $\mathrm{y}=\mathrm{mx}+\mathrm{c}$ can be written in polar form as $\rho=x_{i} \cos \theta+y_{i} \sin \theta$.

Steps for Hough Transform are:

- Lines will be represented as points

- A straight line $\mathrm{y}=\mathrm{ax}+\mathrm{b}$ could be expressed in polar form as $\rho=x_{i} \cos \theta+y_{i} \sin \theta$

- $\quad$ Take one point $\left(x_{i}, y_{i}\right)$.

- Find $\rho=x_{i} \cos \theta+y_{i} \sin \theta$ for various values of $\theta$. 


\section{International Journal of Engineering Applied Sciences and Technology, 2020 \\ Vol. 5, Issue 5, ISSN No. 2455-2143, Pages 122-128 \\ Published Online September 2020 in IJEAST (http://www.ijeast.com)}

- Map those points of $(\rho, \theta)$ as dots in an accumulator cell.

- $\quad$ Repeat this process for all points of $\left(x_{i}, y_{i}\right)$.

- Find the accumulator cell with maximum entries of $(\rho, \theta)$.

- That $(\rho, \theta)$ will give the line with maximum points within it.

$\theta$

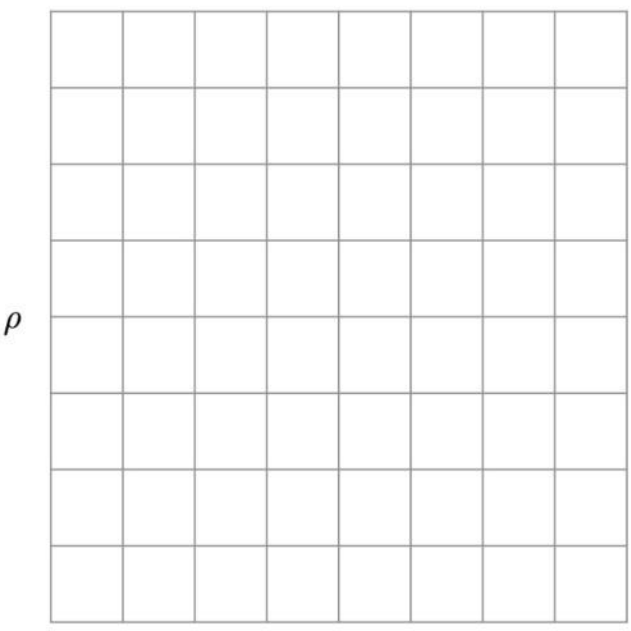

Fig. 5. Hough Accumulator Cells

\section{B. Canny Edge Algorithm}

Canny Edge detector is a multi-stage algorithm to detect a wide range of edges in images. It is the most precise algorithm for detecting edges. It is used in our project for detecting the edges of objects. So, from the images taken using pi camera by canny edge detection process the edges are detected so clearly.

Steps for canny edge algorithm:

- Apply Gaussian filter to smooth the image so as to get rid of the noise.

- A 2D derived function operator is applied to the smoothened image to spotlight regions of image with high spatial derivatives.

- Apply non maximal suppression to remove the spurious response to edge detection. It is mainly applied to thin the sides.

- Apply double threshold (Hysteresis) to determine potential edges.

- Finalize the detection of edges by supressing all the other edges that are weak and not connected to strong edges.

\section{Camera Calibration}

Camera Calibration is the technique used in computer vision applications for estimating the parameters of a camera such as focal length etc. This project uses checkerboard-based camera calibration method. It is the simplest way of calibration under having the enter control over the imaging process. The flow chart given bellow will shows the checkerboard-based calibration method intimately.

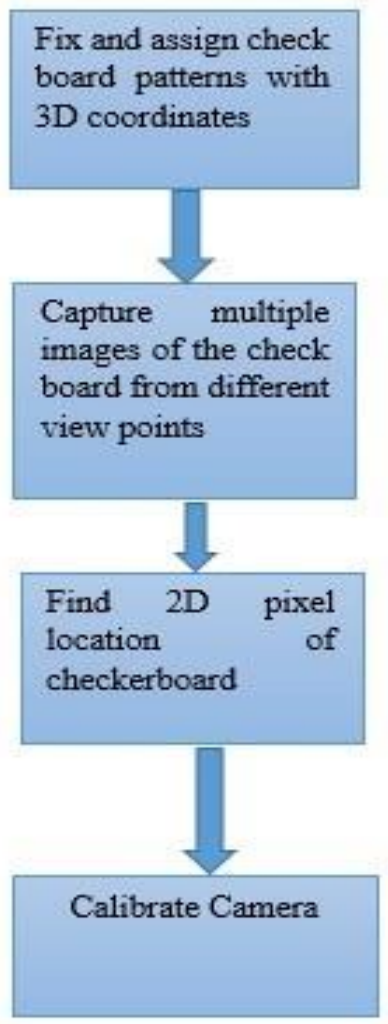

Fig. 6. Flow Chart of Camera Calibration

The primary step in this calibration technique is to fix the checkerboard in 3D coordinates in such a way that all points on the checkerboard are on the $\mathrm{XY}$ plane i.e. $\mathrm{Z}=0$. Since points are equally spaced within the checkerboard, the $(X, Y)$ coordinates of each $3 \mathrm{D}$ point are easily defined by taking one point as reference $(0,0)$ and defining remaining with reference to that point of reference. The next process is to keep the checkerboard static and gather the various images of the board from different angle. After collecting the pictures in order to fix the checkboard corner, use the built-in function of OpenCV called "findChessboardCorners" for getting the coordinates of the corner by checking the board. In order to get good results, use OpenCV's "cornerSubPix" function for obtain the location of corners with subpixel level of accuracy. It takes within the original image, and the location of corners, and looks for the best corner location inside a small neighbourhood of the original location. The ultimate step of calibration is to pass the $3 \mathrm{D}$ points in world coordinates and 
their 2D locations in all images to OpenCV's

"calibrateCamera" method.

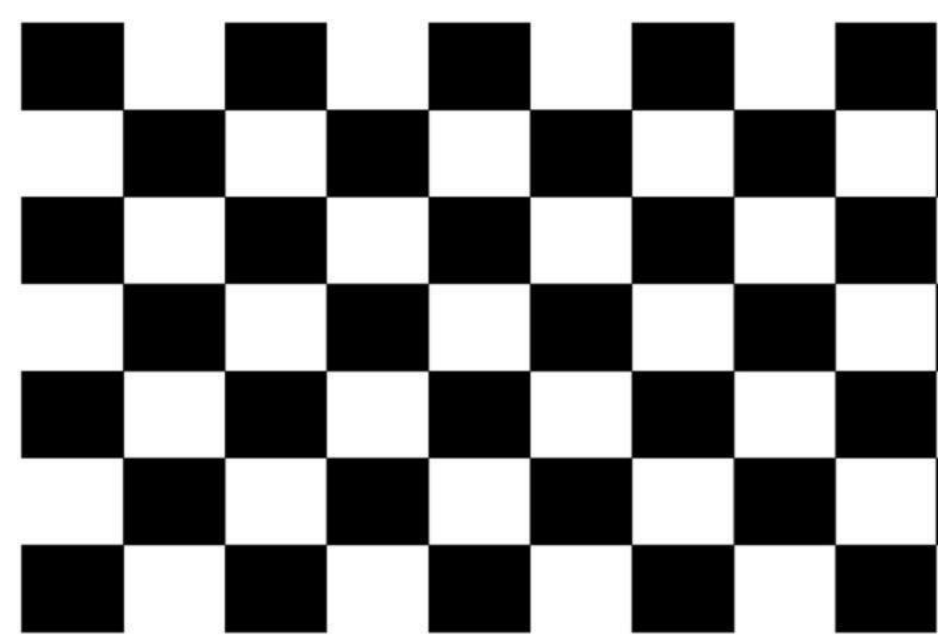

Fig. 7. Image Captured

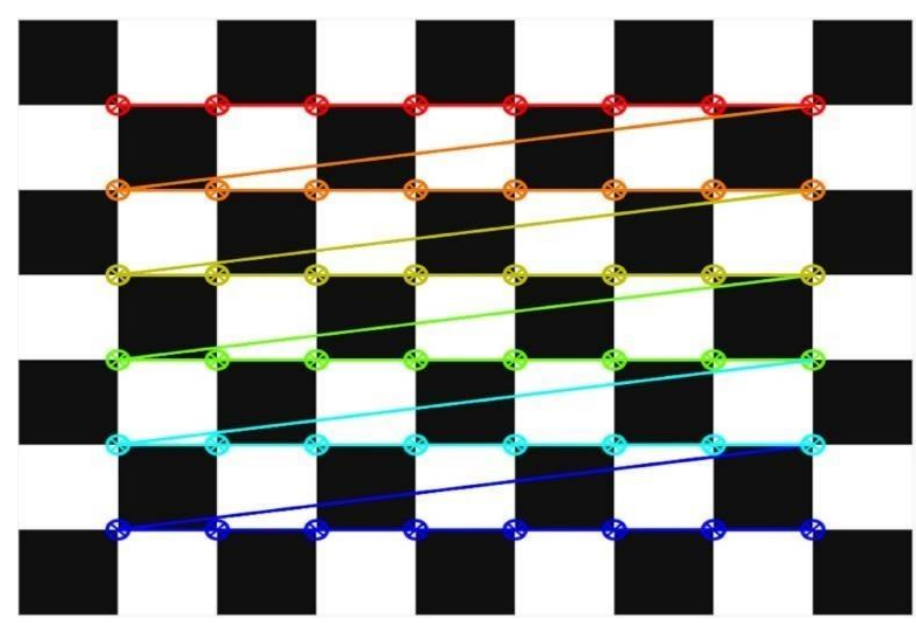

Fig. 8. Result after drawing the detected checker board corners

The pattern size and quality are of utmost important. When we consider the chessboard pattern, the calibration process requires to detect the inner corner of the chessboard and thus the assumption of the algorithm is that every chessboard square is a perfect square.

\section{Line Detection Algorithm}

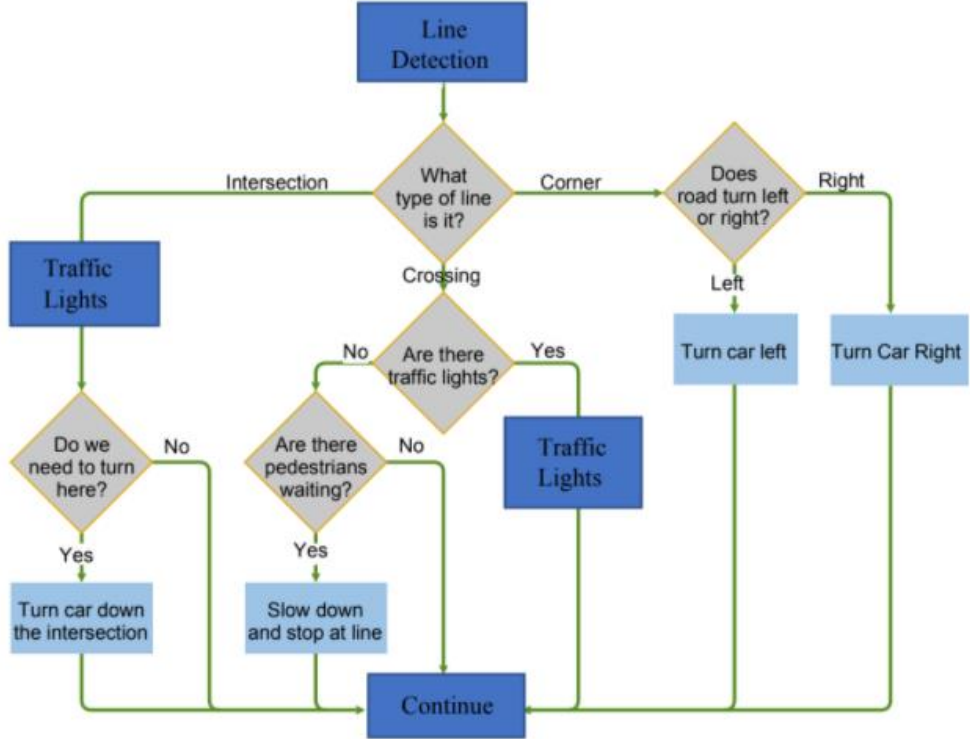

Fig. 9. Line detection Process

In Figure 9, the vehicle will consider the line as intersection line or corner lines. If it is a corner line then it will examine whether it is indicating towards the right side or left side. If it is the left side then it will turn to the left direction else towards the right side. Now examining the reaction towards the intersection lines, during intersection it will give importance to the traffic signal. If the signal is indicating right or left, the conclusion will be made accordingly. Thus, in this traffic signal algorithm comes in action. Now another category that should be considered while line detection algorithm is the crossing lines. Here just like the intersection category traffic signals are considered very importantly. Another matter that is considered is the presence of pedestrian. On the basis of the presence of the pedestrian the vehicle would slowdown or stop.

\section{F. Traffic Signal Detection Algorithm}

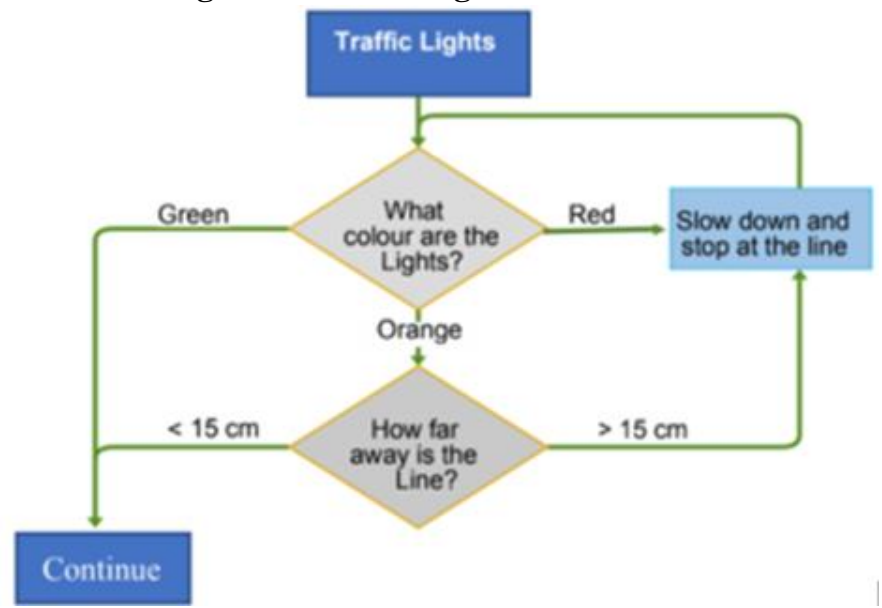

Fig. 10. Traffic Signal Detection Process

Figure 10 shows that as vehicle reaches an intersection, traffic light detection will be activated. Based on the traffic 


\section{International Journal of Engineering Applied Sciences and Technology, 2020 \\ Vol. 5, Issue 5, ISSN No. 2455-2143, Pages 122-128 \\ Published Online September 2020 in IJEAST (http://www.ijeast.com)}

colour displayed the vehicle would decide whether to stop or continue. As the position of lights within the image is known, it is only necessary to process the top part of the image to detect the traffic lights. Colour thresholding is used to determine whether the light is green (go), orange (slow down) or red (stop).

\section{E. Obstacle detection Algorithm}

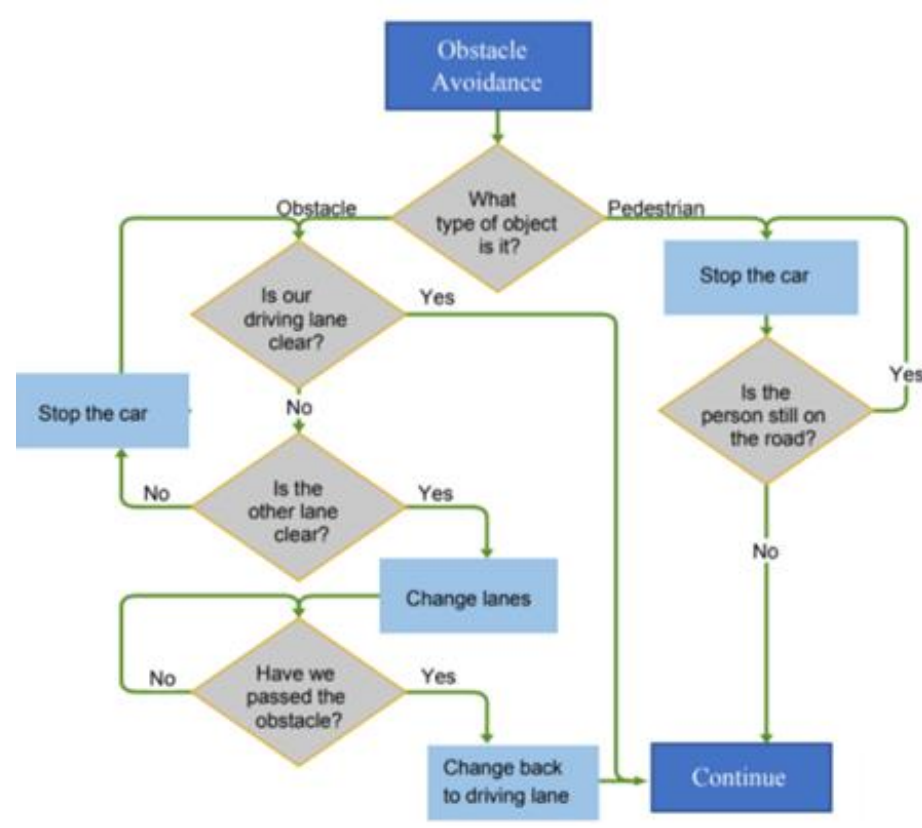

Fig. 11. Obstacle Detection Process

From Figure 11, the system initially checks which type of obstacle is present on the road. It could be vehicles, animals like cat, dog etc or pedestrian who are crossing the road. So first the car interprets whether it is an obstacle or Pedestrian. If it is an obstacle, the system makes sure that the lane is clear and if it is clear, the vehicle will continue to move forward. If it is not clear, it will check whether the other lane is clear or not. If the other lane is clear, the vehicle would be shifted to the next lane. Then it overtakes the obstacle and moves back to the driving lane by keeping a safe distance with the other obstacles. If the other lane is not clear the vehicle automatically stops and re-checks all these conditions and ensures that the driving lane is clear and then proceed to motion.

Now the next case is that of pedestrian detection and avoidance. First it checks whether there is any pedestrian on the moving path or lane. If yes, the car stops. Then it again checks whether the person have moved away or not. If yes, the car starts again to move forward and if not, it re-checks the conditions and waits till the road to be cleared and then continues its motion.

\section{RESULT}

The overall outcome of this project is automatic speed control and object detection which lead to a smooth driving experience for the travelers. The car is trained to move under different paths such as straight track in forward motion.Using Hough Transform the car would track the side lines of the road and using canny edge detection algorithm it would detect the edges. Then the vehicle would move in relative motion with a car in front of it, therefore it provides a safe distance between the vehicles. Next when an obstacle is detected the car gets stopped and restart the motion after the obstacle is cleared and lastly it would follow the traffic signals and sign boards. When the system detects the trained signs, it acts accordigily.

\section{CONCLUSION}

The overall aim of this project is to design an autonomous vehicle which can be used by the senior citizens, differently abled peoples as well as by the one who don't know to drive. The project makes its way through new methodologies and technologies like Computer Vision, Deep learning, that are currently emerging. Computer vison is one among the hottest research field within deep learning at the instant, which represents a relative understanding of visual environments and their contents, this sector paves way towards Artificial General Intelligence because of its cross-domain mastery. Here cameras have been introduced, which comes in action instead of sensor being used in the existing system. Role of cameras, is to obtain input in form of images. Machine/Computer Vision techniques plays a vital role, as a brain does, to interpret the motion of obstacles as well as object near the vehicle which will further help in taking necessary actions like to slow down, apply break etc. As we can see, in this project, the complexity level in the proposed system is comparatively less while compared to sensor-based system. Another added advantage is that the system's capability of identifying traffic lights, and also traffic signals which makes it more reliable for smooth and safe driving. Ensures the smooth working of system with the help of master-slave mechanism.

GPS navigation, extreme weather condition video processing and app interfacing are some of the features which can be further added to the system.

\section{ACKNOWLEDGEMENT}

This project won't be a success without the blessing of almighty and we will always be grateful for this. Now we would like to thank our mentor Mrs. Reeju Eliza Baby who stood with us throughout our project. We are using this occasion to show our gratitude to our ECE department along with our department faculties who always help us regardless 


\section{International Journal of Engineering Applied Sciences and Technology, 2020 \\ Vol. 5, Issue 5, ISSN No. 2455-2143, Pages 122-128 \\ Published Online September 2020 in IJEAST (http://www.ijeast.com)}

of their tight schedule. We would like to convey our gratitude to our Amal Jyothi college for its limitless support along with a wide range of sources which help us to complete our project. Last but not least we would like to convey our gratitude to our family who always stood with us regardless of the inconvenience or situation which played a great role in completing our project.

\section{REFERENCE}

[1] Jain Adithya (2018) on "Working Model of SelfDriving Car Using Convolutional Neural Network, Raspberry $\mathrm{Pi}$ and Arduino" 2nd International Conference on Electronics, communication and Aerospace Technology (ICECA) in 10.1109/ICECA.2018.8474620.

[2] Reddy Poondru (2019) on "Driverless Car-Design of a Parallel and Self-Organizing system." The International Archives of the Photogrammetry, Remote Sensing and Spatial Information Sciences (ISPRS) in Volume XLII2/W7.

[3] Pannu Gurjashan et al (2015) on "Design and Implementation of Autonomous Car Using Raspberry Pi" International Journal of Computer Applications (IJCA) in $\operatorname{Pg}(22-27)$.

[4] A.M. Shah (2018) on "Underwater Image Enhancement by Wavelet decomposition using FPGA" International Research Journal of Engineering and Technology (IRJET) in Volume: 05.

[5] Iqbal Ayesha (2017) on "Design of Multifunctional Autonomous Car using Ultrasonic and Infrared Sensors" Institute of Electrical and Electronics Engineers (IEEE) in 10.1109/ISWSN.2017.8250023.

[6] Heng Lionel (2019) on "Project AutoVision: Localization and 3D Scene Perception for an Autonomous Vehicle with a Multicamera System" International Conference on Robotics and Automation (ICRA) in $\operatorname{Pg}(4695-4702)$.

[7] M. S. Alluhaidan (2019) on "Retinex-Based Framework for Visibility Enhancement During Inclement Weather with Tracking and Estimating Distance of Vehicles" IEEE Jordan International Joint Conference on Electrical Engineering and Information Technology (JEEIT) in 10.1109/JEEIT.2019.8717392.

[8] Amaradi Phanindra et al (2016) on "Lane Following and Obstacle Detection Techniques in Autonomous Driving Vehicles" Institute of Electrical and Electronics Engineers (IEEE) in 10.1109/EIT.2016.7535320.
[9] Khade Kunal et al (2018) on "'Autonomous Electric Vehicle Using Ultrasonic Sensor Skirt Approach" $3^{\text {rd }}$ International Conference for Convergence in Technology(I2CT) in 10.1109/I2CT.2018.8529387.

[10] Jalalat Morteza (2016) on "Vehicle Detection and Speed Estimation Using Cascade Classifier and Subpixel Stereo Matching" Second International Conference on Signal Processing [ICSPIS] in 10.1109/ICSPIS.2016.7869890.

[11] Mukhtar Mashood (2015) on "GPS based Advanced Vehicle Tracking and Vehicle Control System" IJISTA in 10.5815/ijisa.2015.03.01.

[12] Xu Yan' (2018) on "Dual-mode vehicle motion pattern learning for high performance Road traffic anomaly detection " IEEE Conference on Computer Vision and Pattern Recognition (CVPR), in (pp. 145152).

[13] Yuan Yuan (2017) on "An incremental framework for video-based traffic Sign detection, tracking, and recognition" IEEE Transactions on Intelligent Transportation Systems in ( Volume: 18, Issue: 7 ).

[14] Ryan Yee (2018) on "Collaborative Perception for Automated Vehicles Leveraging Vehicle-to-Vehicle Communications" IEEE Intelligent Vehicles Symposium (IV) in 10.1109/IVS.2018.8500388. 